\title{
Fabrication of Hierarchical Structures by Direct Laser Writing and Multi-Beam-Interference
}

\author{
Michael Steger ${ }^{* 1,2}$, Claudia Hartmann ${ }^{* 1,2}$, Stefan Beckemper ${ }^{* 2}$, Jens Holtkamp ${ }^{* 2}$ and Arnold Gillner $^{* 2}$ \\ ${ }^{*}$ Chair for Laser Technology, Technical University Aachen, Steinbachstr. 15, \\ 52074 Aachen, Germany \\ ${ }^{* 2}$ Fraunhofer Institute for Lasertechnology, Steinbachstr. 15, 52074 Aachen, Germany
}

\begin{abstract}
We present a new method for the creation of hierarchical structures by two subsequent steps of laser ablation. To create certain functionalization of surfaces hierarchical structures are often required. These structures can be considered as a microstructure with a superposed nanostructure. Therefore the approach presented in this paper divides the patterning process into two parts: The microstructures are accomplished by direct laser writing with feature sizes down to a few microns whereas the nanostructure is subsequently added by Multi-beam-interference (MBI). Multi-beaminterference is a promising tool for the generation of nanostructures on $3 \mathrm{D}$ surfaces. Within this work the feasibility and limitations of this method are investigated.
\end{abstract}

DOI: $10.2961 / \mathrm{j} 1 \mathrm{mn} .2013 .03 .0005$

Keywords: Direct laser writing, Multi-beam-interference, hierarchical structures, laser structuring, functional surfaces

\section{Introduction}

Micro- and nanostructuring and thus functionalization of surfaces is a key feature for many applications ranging from light guidance devices for consumer electronics to bio-compatibility of implants [1-3]. The wide varieties of functions for these applications range from wettability to optical properties which are mostly inspired by natural surfaces. Hence, mimicking nature has been an important strategy in this field to understand and reproduce the desired functionality. Many research groups were and still are focused on the investigation and recreation of the waterrepellent and self-cleaning ability of superhydrophobic leafs of the lotus plant. The origin of this feature is based on the two-scale surface structure of the lotus leaf. These leafs consist of papillose epidermal cells with a diameter of $\sim 10 \mu \mathrm{m}$ which are superposed by nano-scale tubular wax crystalloids [4]. However, the superhydrophicity can also achieved by one-scale structures which only reproduce either the microstructure or the nanostructure $[5,6]$. The disadvantage of such one-scale structures compared to twoscale structures is a lower contact angle and a reduced stability of the Cassie Baxter wetting regime where the surface acts hydrophobic [4].

Two-scale structures offer a higher stability and selectivity of the desired functionality. The reason for mimicking only one structure is the higher technical effort to generate a nanostructure and the additional barrier of applying it onto a non-plane surface. For the fabrication of nanostructures lithographic processes are commonly used. They allow the reproduction of complex and freely defined structures, but are very cost-intensive due to many complex process steps and high-precision photomasks. Also lithographic processes are limited in regard to useable materials. The processing of 3D-surfaces is difficult or even impossible. Other methods for the generation of nanostructures rely on self-organisation of pure surfaces or an added material under certain environmental conditions. These methods are rather limited in geometry and often lack precise control. Two photon polymerization allows the creation of $3 \mathrm{D}$ structures on the nanometer scale. This process is very time consuming and therefore limited to small areas. In addition only certain materials can be processed.

Multi-Beam-Interference (MBI) has several advantages for the generation of nanostructures [7]: The interference pattern of two or more coherent laser beams can be directly transferred into a surface via laser ablation. These patterns are periodic and their periodicity depends on the beam alignment e.g. the intercepting angle and the laser wavelength. The intensity modulation occurs in the whole volume where the beams intercept. By choosing a symmetrical beam setup, the intensity distribution is constant in the z-plane (see Figure 1) [8]. This ability makes MBI a promising tool for 3D surfaces patterning.

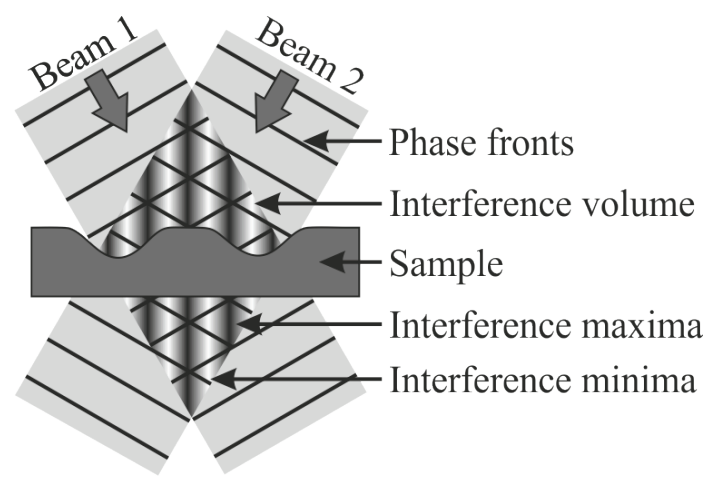

Figure 1: Schematic diagram of an interference volume with two intercepting beams. The sample is shown for illustration purposes only, its influence on the beam propagation is not considered.

In this study we present a method for the generation of hierarchically structured surfaces by two subsequent laser ablation processes. In the first step a microstructure is cre- 
ated by direct laser writing with ultra-short laser pulses (USLP). These patterns are superposed by nanostructures applied by MBI afterwards.

\section{Experimental setup and methods 2.1 Micro Patterning}

The experiments for direct micro patterning are carried out with a diode pumped $\mathrm{Nd}: \mathrm{YVO}_{4}$ MOPA laser (Master Oscillator Power Amplifier; Hyper Rapid, LumeraLaser). The laser operates at a wavelength of $\lambda=355 \mathrm{~nm}$, a maximal repetition rate of $v=1 \mathrm{MHz}$, a gaussian beam shape and a pulse duration of $\tau<15 \mathrm{ps}$. In this work, the repetition rate is set to $v=400 \mathrm{kHz}$.

Due to the design of the implemented Pockels cell, defined pulse bursts can be generated (see Figure 2). Each pulse burst consists of several pulses with an interpulse separation of $\Delta t_{\mathrm{i}}=20 \mathrm{~ns}$. The number of pulses per burst is varied between $n=1$ and 5 .

Due to this strategy of dividing the energy on several pulses in a burst an enhancement of the drilling speed respectively the ablation rate has been shown for the machining for different materials [9-15].
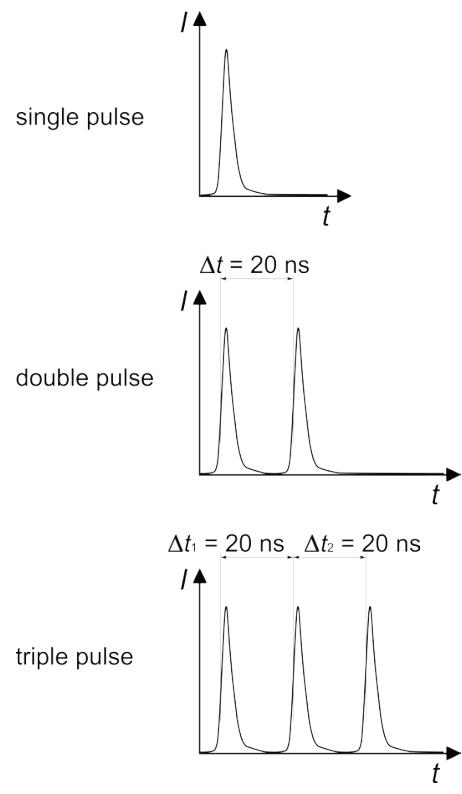

Figure 2: Pulse bursts

The laser beam is deflected by a galvanometric scanner (intelliSCANde ${ }^{\circledR} 14$, SCANLAB) and focused on the surface of the polyimide-foil (PI-foil) by a telecentric $\mathrm{f}-\Theta$-lens with a focal length of $f=32 \mathrm{~mm}$. The ablated geometry is a line pattern.

The ablation parameters (burst energy $E_{\text {burst }}$, pulse ener-

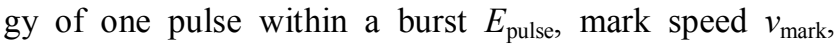
number of pulses per burst $n$ and number of passes $\mathrm{N}$ ) are adjusted to keep the applied energy per length unit constant. The burst energy is varied between $E_{\mathrm{B}}=0.04 \mu \mathrm{J}$ and $1 \mu \mathrm{J}$, the mark speed between $v_{\text {mark }}=100 \mathrm{~mm} / \mathrm{s}$ and $500 \mathrm{~mm} / \mathrm{s}$ and the number of passes between $\mathrm{N}=1$ and 5 .

As ablation results the ablation depth, width and cross sectional area of the lines as well as the angle of the slope are evaluated.

\subsection{Nano Patterning}

The nanostructure is fabricated on unpatterned and micro-patterned samples by Multi-Beam-Interference. The laser beam from a diode pumped Nd:YAG laser (Q301-HD, JDSU) is divided into two equal beams by a $50 \%$ reflective beam splitter and recombined on the sample surface. The laser operates at a wavelength of $\lambda=355 \mathrm{~nm}$ with a pulse duration of $\tau=38 \mathrm{~ns}$, repetition frequency of $v=15 \mathrm{kHz}$, coherence length of $3 \mathrm{~mm}$ and gaussian beam shape. The spatial period of the intensity modulation only depends on the laser wavelength $(\lambda)$ and the angle $(2 \alpha)$ between the intercepting beams:

$$
\Lambda=\frac{\lambda}{2 \sin \alpha}
$$

The spatial period is varied by adjusting the intercepting angle. For further control of beam parameters a spatial room filter and a telescope are integrated in the optical path. For each beam a system of two half-wave plates and a glan-prism are installed to ensure an optimal polarization perpendicular to the plane of the intercepting beams and an equal amplitude of each beam (see Figure 3 ).

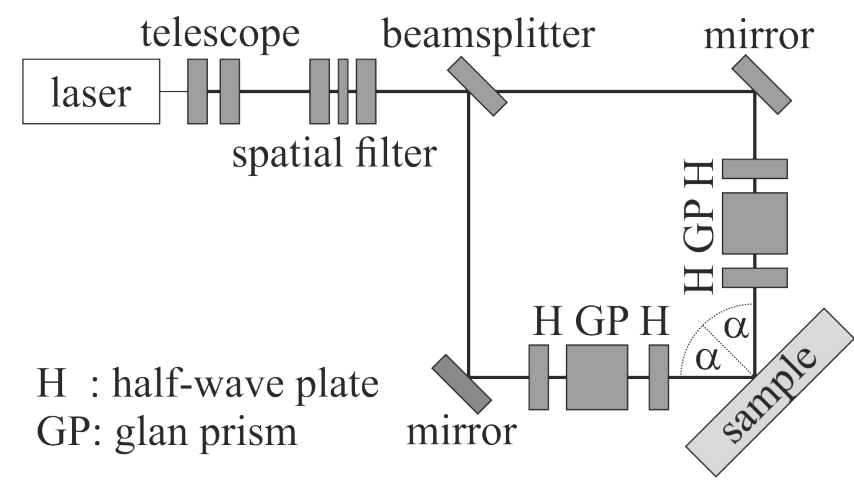

Figure 3: Experimental 2-beam interference setup $\square$

The intensity modulation created by the interference is present in the whole volume where the beams intercept. For a two beam interference the resulting intensity modulation is:

$$
I(x, y)=2 \mathrm{I}_{0} \cos (k x \sin (2 \alpha))^{2}
$$

where $\mathrm{x}$ and $\mathrm{y}$ are the lateral coordinates, $\mathrm{I}_{0}$ the energy density of both laser beams and $k$ the wave number. This energy distribution (2) results in a line-wise modulation with constant values along the y-axis. Furthermore the position of maxima und minima stays constant along the $\mathrm{z}$-axis within the interference volume which allows the patterning of non-plane surfaces (see Figure 1). With a beam diameter of $950 \mu \mathrm{m}$ this volume has a height of $5.3 \mathrm{~mm}$ to $2.3 \mathrm{~mm}$ depending on the beam alignment. The angle between the beams is varied from 10.2 to 24.4 degree.

This study is focused on the creation of hierarchical structures consisting of a microstructure and a nanostructure. The interaction with the microstructure during the nano-patterning process and the general feasibility of this approach are therefore the subject matter. To avoid an influence of other nanostructures which are not a consequence from the micro patterning (e.g. ripples) only single pulse experiments are carried out. 


\subsection{Sample material and characterization}

For the experiments polyimide foil (Upilex) with a thickness of $50 \mu \mathrm{m}$ was used. Prior to the structuring steps the sample material was cleaned with pure water in an ultrasonic bath.

The patterned surfaces are investigated by confocal laser-scanning-microscopy (LSM, VK-9700, Keyence) and atomic force microscopy (AFM, Rados N8, Bruker). While LSM is sufficient for measurement of microstructures and detection of nanostructures, the AFM is necessary for a precise measurement of the nanostructure, but it is limited by its $\mathrm{z}$-shift.

\section{Results and Discussion}

\subsection{Micro Patterning}

The micro patterning experiments have the goal to generate structures with feature sizes smaller than $10 \mu \mathrm{m}$ and an adjustable slope. To achieve small structure sizes the pulse energy has to be as small as possible. With decreasing pulse energy the structure size, mainly the width can be reduced (see Figure 4). In order to achieve a stable ablation process, the pulse energy must not go below a minimal value of $0.067 \mu \mathrm{J}$. The slope which can be reached is $\gamma=$ $57^{\circ}$ at the highest (see Figure 4).

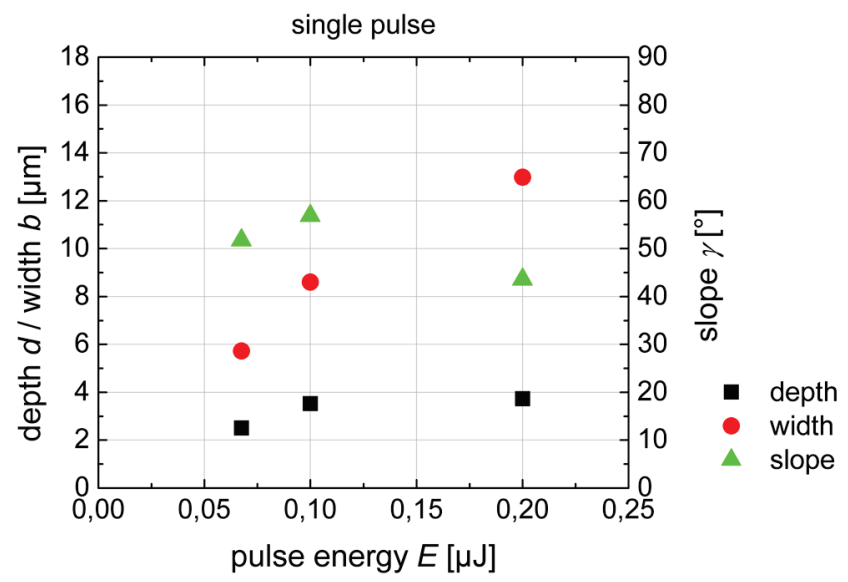

Figure 4: Ablation depth, width and slope of the line structures generated with single pulse ablation. $(v=100 \mathrm{~mm} / \mathrm{s}, v=400 \mathrm{kHz}$,

$$
\mathrm{n}=1 \text { to } 5 \text { ) }
$$

For variation of shape of the ablated geometry as well as for increasing the ablation speed, pulse bursts have been previously used.

When dividing the energy of one burst into several pulses different results have been shown for the ablation with UV ps-bursts $[14,16]$ both with and without increase of the ablation rate. As for line patterning with pulse bursts many parameters can be changed. To be able to compare the results with each other, two different sets of parameters are investigated here:

- constant burst energy:

Each burst comprises the same total energy as the single pulse. (The scan speed is kept constant)

- constant energy per pulse

Each pulse within the burst has the same energy as the single pulse. (The scan speed has to be increased to keep the energy per line constant)
When dividing the energy of the burst on the pulses of the burst the energy of each single pulse of the burst gets smaller. The ablation using pulse bursts can still be performed with smaller energy per pulse than when using single pulse ablation (see Figure 5). A stable ablation process still takes place for a five pulse burst where each pulse comprises $40 \mathrm{~nJ}$. At this pulse energy no stable ablation process can be achieved using single pulses where the minimal pulse energy is $67 \mathrm{~nJ}$ (see Figure 4).

Using pulse bursts the ablation depth increases with increasing number of pulses per burst and with that decreasing energy per pulse. This is contrary to the ablation with single pulses. Here the ablation depth decreases with decreasing pulse energy. The width decreases in both cases with decreasing pulse energy (res. increasing number of pulses per burst) (see Figure 4 and Figure 5).

The slope of the structures is increased by using bursts instead of single pulses. A maximal slope of $\gamma=75^{\circ}$ is achieved (see Figure 5).

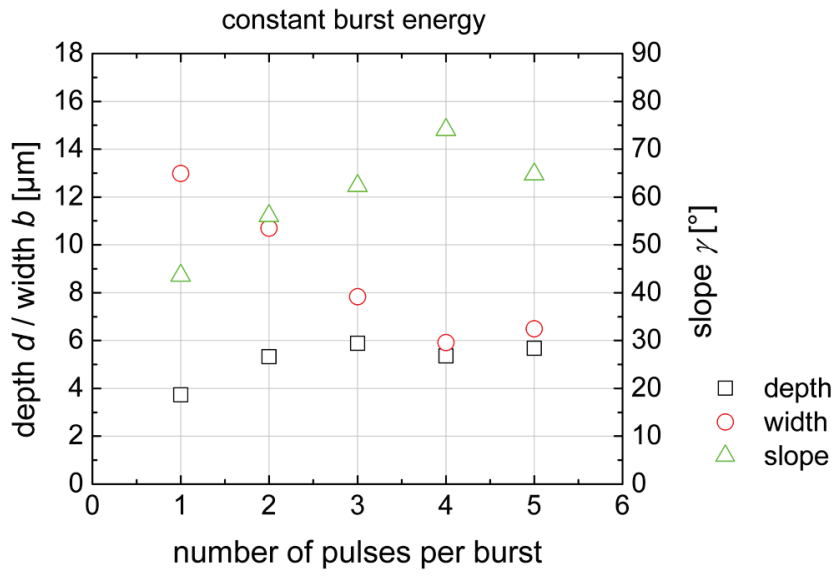

Figure 5: Ablation depth, width and slope of the line structures generated with pulse burst ablation. The total energy in the pulse burst is kept constant. $\left(E_{\text {Burst }}=0,2 \mu \mathrm{J}, v=100 \mathrm{~mm} / \mathrm{s}, v=\right.$ $400 \mathrm{kHz}, \mathrm{n}=1)$

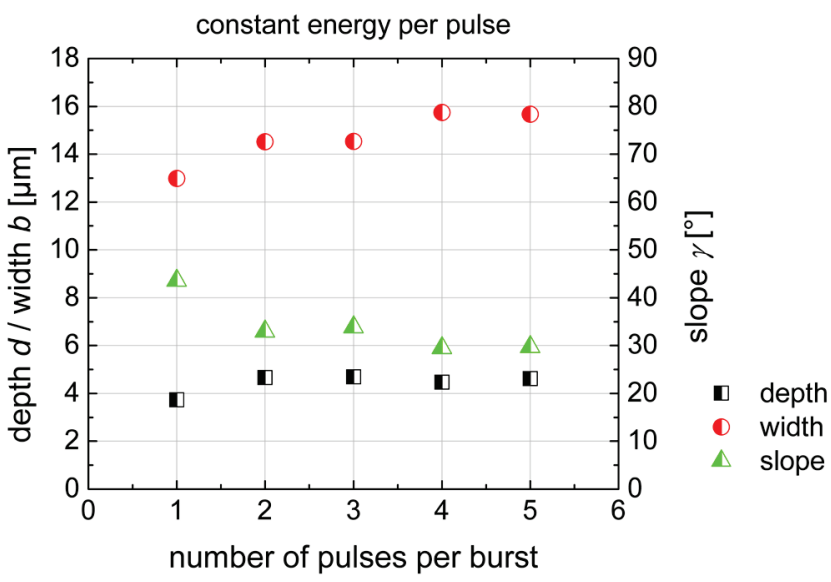

Figure 6: Ablation depth, width and slope of the line structures generated with pulse burst ablation. The energy per pulse in the burst is kept constant. ( $E_{\text {Burst }}=0,2$ to $1 \mu \mathrm{J}, v=100$ to $500 \mathrm{~mm} / \mathrm{s}, v$ $=400 \mathrm{kHz}, \mathrm{n}=1)$ 
When keeping the energy per pulse constant within the burst, the burst energy increases with increasing number of pulses per burst. To keep the overall energy per line constant the scan speed is increased.

The depth of the ablated line is nearly constant when increasing the number of pulses per burst. The width of the line increases drastically with increasing numbers of pulses per burst (see Figure 6). The slope of the lines decreases to values of $\gamma \approx 30^{\circ}$ (see Figure 6).

For comparison of the amount of the ablated material, the cross sectional area of the ablated lines is evaluated.

The cross sectional area increases with increasing energy when using the single pulse ablation (see Figure 7). Using pulse burst ablation with constant energy per pulse, the cross sectional area also increases with increasing energy per pulse. It only is smaller for single pulse ablation (see Figure 7). Due to pulse bursts, the amount of ablated material can be more than tripled. In comparison with the ablation using single pulses of $70 \mathrm{~nJ}$ pulse energy, the burst energy needed for the ablation with triple pulse bursts decreases to $0,2 \mu \mathrm{J}$ (see Figure 7).

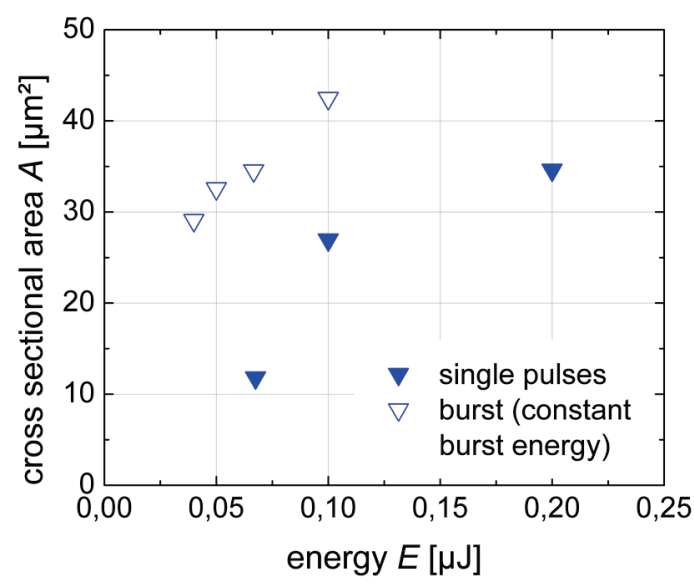

Figure 7: Cross sectional area of the ablated lines for comparison of single pulse ablation (compare Figure 4) and burst abla-

tion at constant energy for each pulse (compare Figure 5).

When comparing the ablation with pulse bursts with the different parameters used (constant burst energy and constant energy of each pulse within the burst) different results are achieved. When using constant burst energy the cross sectional area is maximized for double pulse bursts and reduced with increasing numbers of pulses per burst (see Figure 7). As each pulse contains less energy the more pulses are within one burst, this energy gets closer to the ablation threshold and therefore less material is removed.

Using constant energy per pulse within the burst the cross sectional area increases with increasing number of pulses per burst (see Figure 8). Due to the short spatial distance between the single pulses within the burst, the material removal benefits from thermal effects.

The greatest improvement of burst ablation is achieved in the overall ablation speed. The amount of ablated material per time is increased by a factor of more than 6 by using bursts with five pulses with constant energy per pulse in comparison to single pulses at the same pulse energy. The cross sectional area is increased by a factor of 1.3. The ablation speed is increased by a factor of 5 . Therefore burst ablation has the potential to increase the ablation speed dramatically.

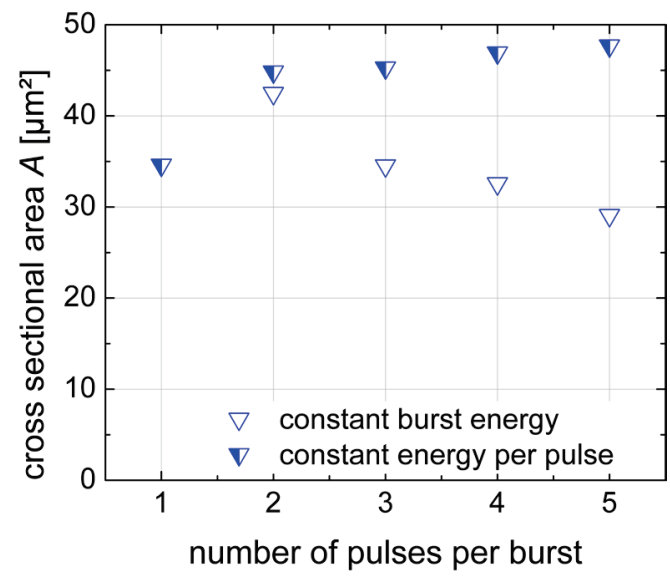

Figure 8: Cross sectional area of the ablated lines for comparison of burst ablation with constant burst energy (compare Figure

5) resp. constant energy for each pulse (compare Figure 6).

\subsection{Nano Patterning}

We investigate the patterning process by MBI first on plane samples surfaces. The aim of these experiments is to identify parameter sets with a maximized structure depth for the later application on hierarchical structures and a general study of the created structures.

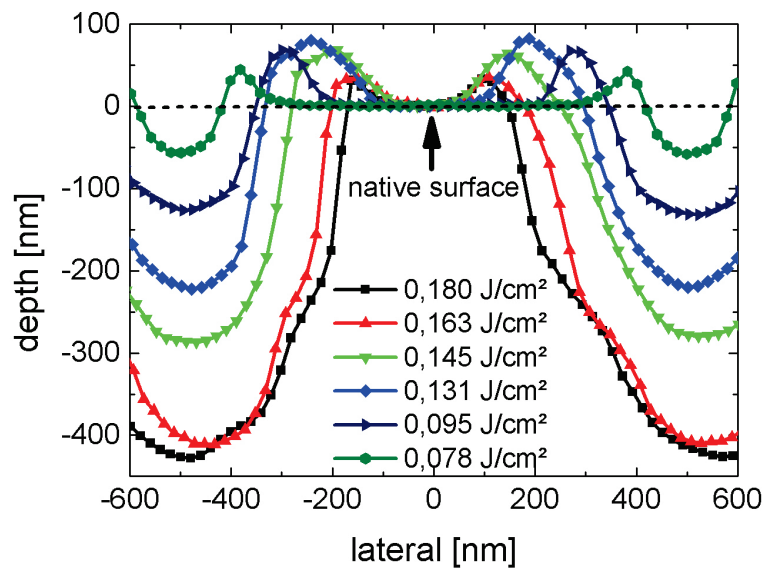

Figure 9: Cross sectional area of grooves generated by 2-beaminterference with a periodicity of $1 \mu \mathrm{m}$.

As shown in Figure 9 the laser fluence can be used to adjust the depth and width of the structure inside a unit cell of the chosen periodicity. In this figure it shown exemplarily for a periodicity of $1 \mu \mathrm{m}$. For the other periodicities the behavior is similar and the maximal depth of the structures is stated in Table 1. All fluence values are average values over the temporal and spatial beam shape. For lower fluence the width and depth of grooves decreases. For single pulse experiments these parameters cannot be varied independently. The ablation threshold is $F_{\text {th }}=0.039 \mathrm{~J} / \mathrm{cm}^{2}$. No significant variation for different periodicities can be detected. The upper fluence limit before a collapse of the structure occurs varies depending on the periodicity. A decrease in the periodicity reduces the maximal applicable 
fluence. Above this critical fluence damages appear (see Figure 10). If the fluence is further increased, the remaining bridges between the grooves get detached from the surface. The damage occurring slightly above the critical fluence shows an interesting behavior: The remaining bridges seem to collapse to a side and tend to form pairs instead of a statistical distribution. The cause of this effect is not identified yet and needs further investigation.

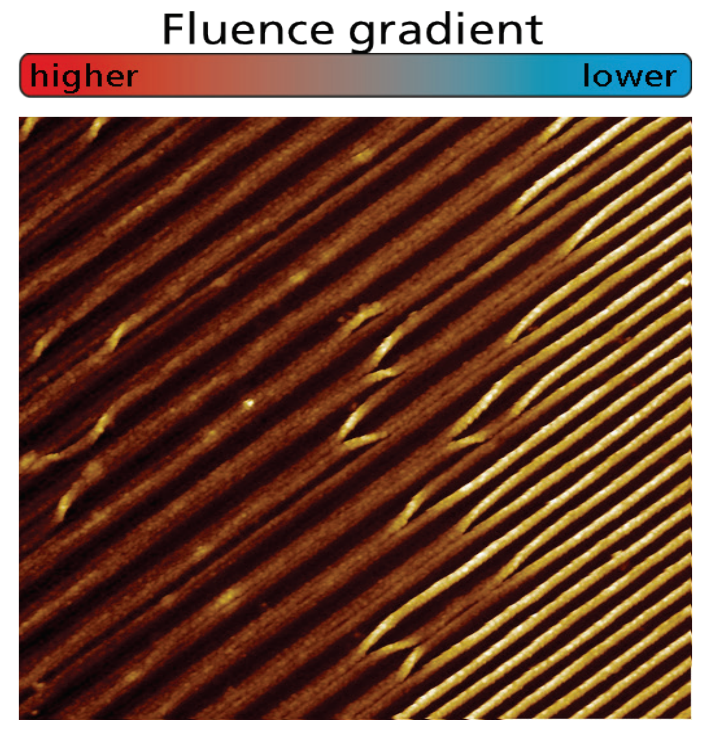

Figure 10: AFM measurement of area with fluence gradient. The scan size is $20 \mu \mathrm{m} \times 20 \mu \mathrm{m}$.

The ideal fluence for generating nanogrooves with maximized depth by a single pulse patterning at four different periodicities is identified (see Table 1). With the exception of $1 \mu \mathrm{m}$ this value also marks the maximal fluence before a degradation of the structures occurs.

Table 1 Maximal structure depth for different periodicities

\begin{tabular}{ccc}
\hline Periodicity & Fluence & Depth \\
\hline $1000 \mathrm{~nm}$ & $0.180 \mathrm{~J} / \mathrm{cm}^{2}$ & $440 \mathrm{~nm}$ \\
$750 \mathrm{~nm}$ & $0.095 \mathrm{~J} / \mathrm{cm}^{2}$ & $350 \mathrm{~nm}$ \\
$500 \mathrm{~nm}$ & $0.068 \mathrm{~J} / \mathrm{cm}^{2}$ & $270 \mathrm{~nm}$ \\
$430 \mathrm{~nm}$ & $0.061 \mathrm{~J} / \mathrm{cm}^{2}$ & $125 \mathrm{~nm}$ \\
\hline
\end{tabular}

\subsection{Hierarchical structures}

As the final set of experiments the nanostructures created by MBI are applied onto different micro-structured surfaces. A result from two separate nano structuring steps with a periodicity of $1 \mu \mathrm{m}$ is presented in Figure 11 . The sample is turned by $90^{\circ}$ between the two MBI irradiation steps to generate a rectangular bump structure. A micro structure with a depth of $4 \mu \mathrm{m}$ is chosen for this experiment. The nanostructure superposes the microstructure completely. The geometry and depth of the bumps differ locally depending on the slope angle of the microstructure due to the changing fluence. From the ideal quadratic bump shape on plane surface elements, the bumps are distorted to rectangular shapes with increasing slope angle. An increasing angle of the slope also reduces the depth. On relatively plane elements of the microstructure at the top of the ridge and at the bottom the depth is similar to experiments on unstructured surfaces. Furthermore, it should be noted that nanogrooves which form the bumps are parallel to each other and are not necessarily perpendicular to the surface.

With a higher slope angle the previously described deviations of width and depth of the structures increases until the local fluence falls below the ablation threshold. In Figure 12 nano structures with different periodicity of a microstructure with a depth of $5.2 \mu \mathrm{m}$ and slope of $42^{\circ}$ is shown. While the nano structures is clearly visible with a periodicity of $1 \mu \mathrm{m}$ and $750 \mathrm{~nm}$, the added structure (periodicity of 500 and $430 \mathrm{~nm}$ ) seem to be incomplete or undetectably shallow at the flanks of the micro structure. Other deviations from the expected structures could not be observed on any of the structured samples. These deviations can be for example a consequence from reflections or diffractions at the microstructure disturbing the interference pattern.

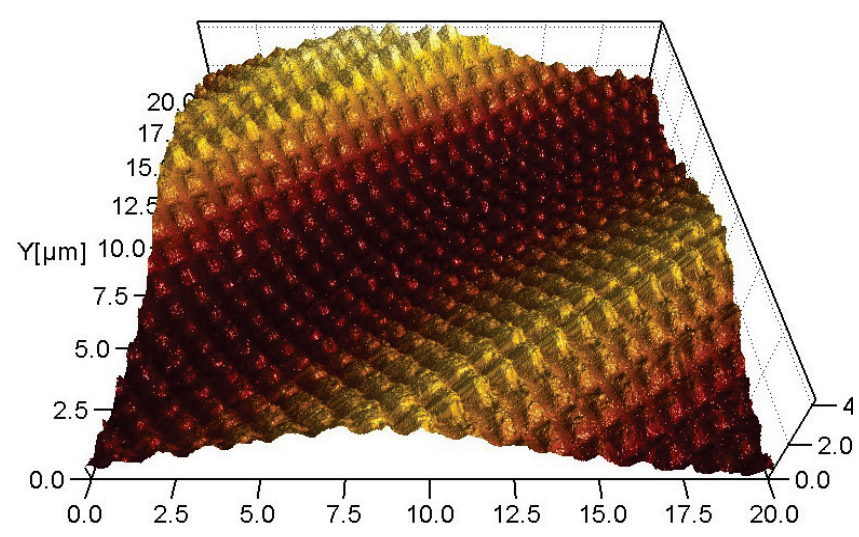

Figure 11: AFM measurement of a nanostructure processed two times with an angle of $90^{\circ}$ between the nanostructuring steps. The three axis are scaled $1: 1: 1$
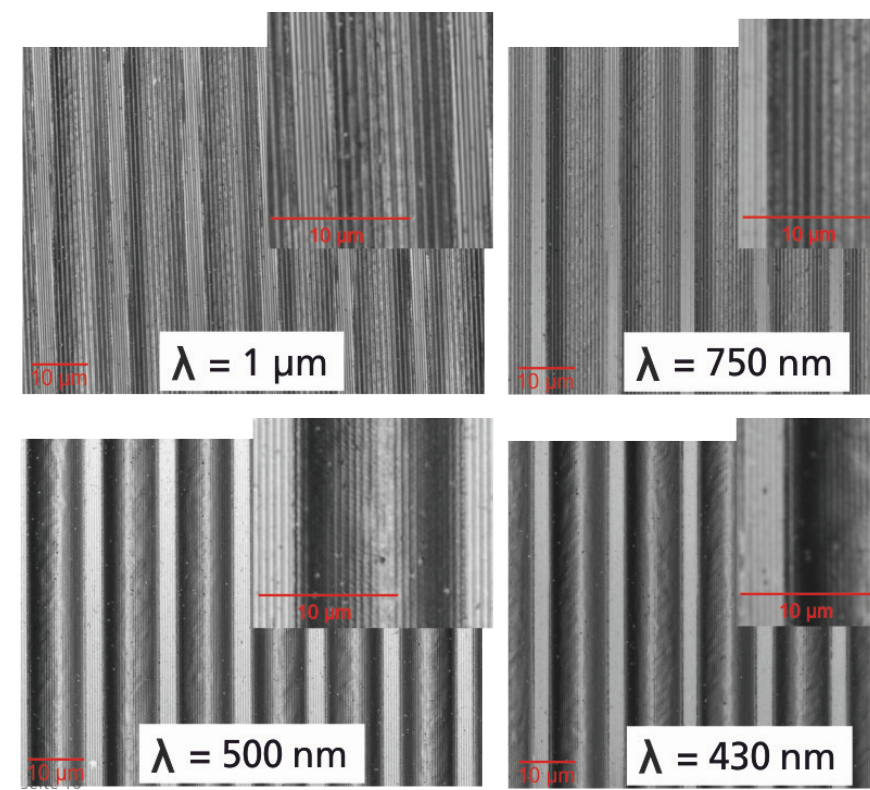

Figure 12: LSM measurement of micro structure with depth 5.2 $\mu \mathrm{m}$ and angle of $42^{\circ}$ which is superposed by 4 different periodicities $(\Lambda)$ of nano structures. 


\section{Conclusions}

The generation of hierarchical structures via two subsequent laser ablation steps is a feasible method to generate well-defined microstructures with superposed nanostructures. Based on the results, the following conclusion can be drawn:

- Micro patterning with ultra-short pulse bursts allows the creation of smaller and deeper structures compared to single pulse ablation. The ablation threshold is reduced for ultra-short pulse bursts which leads to a more precise ablation process. Additionally the amount of ablated material per time is increased by a factor of more than 6 by using burst with five pulses at constant energy per pulse in comparison to single pulses at same constant energy per pulse.

- Multi-beam-interference can be applied to generate periodic nanostructures in one ablation step simultaneously over the whole surface plane inside the interference volume. The structure size ranges from $100 \mathrm{~nm}$ to the micron regime. With a decrease of periodicity of the structures the process windows is also reduced and the achieved aspect ratio of the structures is lower.

- The fabrication of hierarchical structures by subsequent direct laser writing and multi-beaminterference is possible, but limited due to fluence variations at the slopes of the microstructure. This leads to different depths and widths of the generated nanostructures on surface elements.

\section{Acknowledgments}

Parts of the here depicted research was funded by the German Research Foundation (DFG) as part of the Cluster of Excellence "Integrative Production Technology for HighWage Countries" and as part of the DFG priority program "Generation of sub-100 nm structures by nonlinear lasermaterial interactions".

\section{References}

[1] SH. Baik, SK. Hwang, YG. Kim, GJ. Park, JH. Kwon, WT. Moon, SH. Kim, BK. Kim, SH. Kang: Journal of the Optical Society of Korea, 13, (2009), pp. 478-483

[2] E. Bremus-Koebberling, S. Beckemper, B. Koch and A. Gillner: Journal of Laser Applications, 24, (2012)

[3] D. Langheinrich et al: Journal of Polymer Science Part B: Polymer Physics, 50, (2012), pp. 415-422

[4] K. Koch et al: Soft Matter, 5, (2009), pp. 1386-1393

[5] PF. Hai, ZH. Yaia and XW. Zhang: Science China: Physics, Mechanics \& Astronomy, 54, pp. 675-682

[6] KC. Park et al: ACS Nano, 6, (2012)

[7] D. Xia et al: Advanced Materials, 23, (2011), pp. 147179

[8] L.Z. Cai, X.L. Yang and Y.R. Wang: Journal of Modern optics, 49, (2002), pp. 1663-167

[9] R. Stoian, M. Boyle, A. Thoss, A. Rosenfeld, G. Korn, and I.V. Hertel: Appl. Phys. A 77, 265, (2003).

[10]M. Lapczyna, K.P. Chen, P.R. Herman, H.W. Tan, and R.S. Marjoribanks: Proceedings of the 5th International Conference on Laser Ablation, Göttingen (1999), pp.883-886.

[11]P.R. Hermann, A. Oettl, K.P. Chen, and R.S. Marjoribanks: Proceedings of the Conference on Commercial and Biomedical Applications of Ultrafast Lasers, San Jose, California (1999), pp.148-155.

[12]A.C. Forsman, P.S. Banks, M.D. Perry, E.M. Campbell, A.L. Dodell, and M.S. Armas: J. Appl. Phys. 98, 033302 (2005).

[13]B.R. Campbell, T.M. Lehecka, V.V. Semak and J.G. Thomas: Proceedings of the 26th International Congress on Applications of Lasers and Electro-Optics, Orlando (2007), pp.45-51.

[14]C. Hartmann, T. Fehr, M. Brajdic, and A. Gillner, Journal of Laser Micro/Nanoengineering 2, (2007), pp. 44-48

[15]K. Bobzin, N. Bagcivan, M. Ewering, A. Gillner, S. Beckemper, C. Hartmann, S.Theiß., Journal of Nanoscience and Nanotechnology 11, (2011), pp. 87758781

(Received: August 27, 2013, Accepted: November 6, 2013) 